\title{
Electromagnetic Protection of Airborne Data Transmission System
}

\author{
Zhenwei Chen \\ Military sales department of Shenyang Aircraft Corporation Post Code: 110850
}

\begin{abstract}
The data transmission of aircraft data link is always interfered by other electronics or electrical equipments, which easily cause a glittering array of data error and effect the normal operation of airborne products. This paper analyzes possible electromagnetic interference of the airborne data system and introduces various electromagnetic protection approaches.
\end{abstract}

Keywords-Electromagnetic compatibility; Shielding; Rejection interfering

\section{INTRODUCTION}

The airborne data transmission system is adopted in the transmission between aircraft and aircraft as well as ground-control station and data system products are distributed in various parts of the aircraft. In addition, the rapid development of digital technology as well as the diverse and distributed development of data transmission requires improvement in terms of aircraft capacity, emission and receiving sensitivity. Therefore, the design develops toward the trend of integration and modularization which requires higher transmission intensity and demand in terms of electromagnetic interference environment. In modern wars, the electromagnet interference encourages us to strengthen the electromagnetic compatibility protection so as to guarantee that the data transmission is smooth and reliable.

Electromagnetic compatibility refers to the capability of two or more electrical devices to operate simultaneously without interference. The airborne data transmission has powerful launching capacity as well as high receiving sensitivity, which is more useful in terms of electromagnet interference comparing to other electronic equipments. Therefore, the magnetic compatible protective measures can be regarded as the basis for products normal operation. This paper mainly elaborates the electromagnetic protection transmit-receive approaches of data transmission system.

\section{DATA TRANSMISSION SYSTEM GROUND CONNECTING DESIGN}

Electromagnet interference puts the wireless signal to the internal data system by wire, which cannot be easily recognized because the invisibility ability is good. Besides, the current of wire returns to each electric circuit and if there should be improper ground wire setting of the receiver, the electromagnet interference is easily introduced and the products cannot work in a normal state.

\section{A. Ground connecting protection approaches}

The ground connecting is to provide a potentiometric point or surface for the circuit or system, which also provides low-impedance path. Besides, the basic principle for receiver data system's wire design is that the ground wire length should be shorter than one-twentieth of the shortest wavelength.

Measures show as follows:

The cabinet, shield case and ground wire should be closely integrated so as to reduce the contact resistance and connecting inductance.

In circuit setting, large-scale ground connecting is proper and the printing plate ground wire should be widened so as to reduce the inductance as well as the characteristic impedance.

The lay, decoupling measures and power supply wiring should be comprehensively taken into consideration so as to guarantee that the alternating component is connected to the closest ground with the shortest distance returning to the circuit.

Properly lay ground wire so as to guarantee that the current has each circuit to avoid interaction and interconnection as well as closed-loop circuit.

\section{B. Ground connecting forms and approaches}

There are three form grounds, which are analog signal, digital signal and noise ground. The circuit cheater of receiver and its power supply belong to the analog signal; the digital circuit and its power supply belongs to digital signal ground while the transformer of alternating current power supply and shield ground of AC power line belong to noise ground. These three are strictly distinguished to reduce the interference of ground wire.

The ground connecting for data system can be divided into the following:

The suspension refers to the ground wire of electronic equipments as well as the surrounding insulation, yet the crosstalk interconnection interfere the equipment. The signal of this equipment connects with other circuits and systems via cables. Besides, the signal connecting with other circuit and ground via outer conductor of cable is used to protect special electric circuit, showing in Fig. 1. Single point earth contents with each other based on 
common reference without problems such as impedance coupling and low-frequency ground loop, showing in Fig. 2. Multipoint earth (f>10MHz) has each annunciator wire which may easily cause ground loop. The transient earth voltage will be overlaid in useful signals which can cause interference. The suppressing means is to cut off the ground loop. While adopting multipoint earth, common electric circuit takes cabinet as the reference point and therefore we should try our utmost to reduce the resistance and inductance.

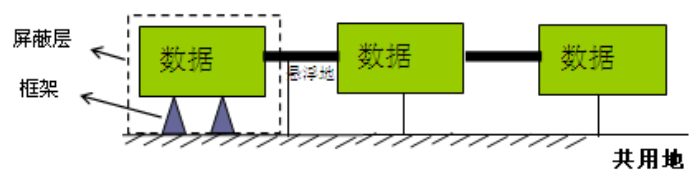

Figure 1. Suspension

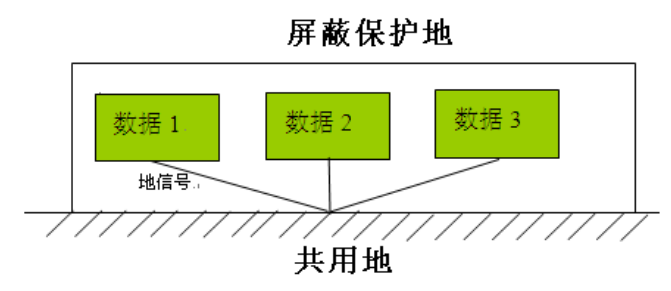

Figure 2. single point earth

Multipoint earth shows in Fig. 3

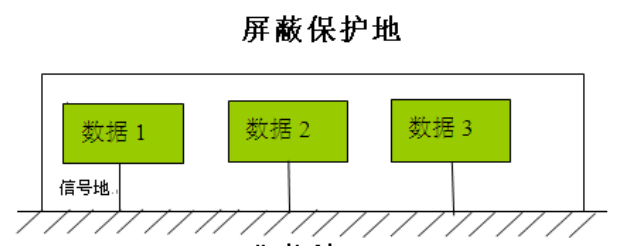

公共地

Figure 3. Multipoint earth

In receiver system design, microwave circuit, intermediate-frequency circuit cheater and high-speed digital circuit always adopt large-scale multipoint earth; the connecting mode between module and module as well as digital circuit boards is single point earth; key protected electronic circuit such as reference oscillator can adopt suspension.

In certain research project work, the small local space leads to chaos of equipment earthing cable. Besides, the loop current in ground test equipment runs through the ground wire of airborne station, triggering the interference of airborne station, which only can be solved by changing ground location and treatment of various ground wires. It indicates that the ground wire design, to some extent, affects the airborne station operation.

\section{SHIELDING APPROACHES FOR DATA SYSTEM}

The shielding approaches are adopted to prevent the delivery of electromagnet interference. Besides, proper strengthening the shielding coverage as well as the reliability can help to reduce the fluctuation of data signal transmission.

\section{A. $\quad$ Shielding design of data system}

In electromagnetic compatibility design, shielding design is of great significance. Shielding is an important means to reject any irrelevant signals which can be divided into three types: static shielding, magnetic shielding, and electromagnetic shielding. In the space, any two charged objects shall generate electrostatic field and the voltage change of one side will lead to the change of the other side and generate electrostatic coupling. The best approach to overcome electrostatic coupling is to adopt metal plate as the shielding body so as to separate them. The important thing is that the shielding should be perfectly connected to the ground or it won't generate shielding function.

The current flows in the wire and magnetic field is created which responds to each other based on mutual inductance. Especially in low frequency condition (under $3 \mathrm{KHz}$ ) the main interference is caused by the magnetic field. The electromagnetic shielding is related to the nature, change frequency of electromagnetic field as well as the distance between radiation source and sensor. The receiver's electric system with electronic circuit above $10 \mathrm{KHz}$ always adopts aluminium as the electromagnetic interference shielding material which can shield $100 \mathrm{~dB}$ in terms of the $300 \mathrm{MHz}$ signal. At the same time, in the receiver electrical system, the shielded box is always equipped with blind flange for convenience. What's more, there are open holes and seams for ventilation and heat dispersion which will cause discontinuity points and lead to leakage of signals and interference. Therefore, we have to carefully consider the proper setting of components so as to guarantee that the open holes and seams won't cut off the induced current. If necessary, the open holes can be changed with plumbing so as to reduce the radiation effect.

\section{B. The shielding approach for the data system}

Except for shielding design, the packaging technique is also of great significance, especially the RF system packaging.

Precautions for shielding design: the shielding design for internal electric circuit should be able to prevent the leakage of RF energy and at the same time can prevent the external electromagnetic energy effect. Besides, we should also prevent the unnecessary feedback and coupling among circuits. In addition, if necessary, absorbing material can be added to absorb the feedback energy. Furthermore, we can carry out decouple for the power supply filter to prevent or decay the internal transmission or the transmission among electronic 
portfolio. What is worth paying attention to is that the smaller the inductance, the better effect the radio frequency ground resistance.

Shielding box design: if there should be higher requirement for data transmission attenuation we have to use better box and build it into single isolated room or various rooms, namely a box with various rooms, which will cause good isolation effect for electrostatic and electromagnetic fields. Besides, the manufacturing box should adopt aluminum, which is cheap and light with good isolation effect for the electrical system. While decorating the shield, leakages always occur and we have to take necessary actions to make more contacts. Besides, we can add nut bolt as well as flexure strip and electric gasket to prevent anticorrosion especially the corrosion or we cannot have good effect.

Radio frequency transmission line design should solve the RF interference and we have to properly choose amplitude of transmission signal so as to correctly arrange cable louding. Besides, the pulse signal line as well as the communication annunciator wire should not be integrated. Besides, significant signal impulse should be strictly distinguished from the high purity signal. Shielding design is a complicated task and we have to firstly be familiar with the knowledge of circuits, magnetic and electromagnetic fields. Meanwhile, we should be aware of the knowledge in terms of electrical construction and process design.

In early times, airborne station has good capability in ground test and will be interfered in demonstration and the reason is that the shielding ability is not good enough. Nowadays, most of the airborne stations adopt various shielding technology which are seldom interrupted by

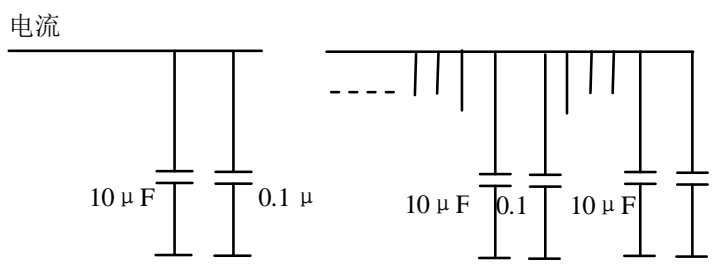

other electronics or electronic equipments because of the shielding ability.

\section{DATA TRANSMISSION SYSTEM ELECTROMAGNETIC} COMPATIBILITY

\section{A. Electromagnetic compatibility design in data} transmission system

In electromagnetic compatibility testing, the interference comes from power sully will also affect the data transmission.

The data signal coming from the external power source cannot only be introduced by AC power cord but also electromagnet interference; the power supply is adopted for various electric circuits and the current alterations will cause voltage depression on internal resistance, which is then transferred to other electric circuits and lead to mutual interference.

Suppressing measures: Shielding measures for mains transformer

Power transformer shielding: static shielding should be added to the first-level winding so as to reduce the interference and the whole transformer should be magnetically shielded to prevent the interference brought by transformer of magnetic field. All these shielding should be perfectly connected to the ground. Filtering and decoupling: each circuit system as well as printed circuit should be filtered and decoupled which is made up of a couple of $\mu \mathrm{f}$ electrolytic condenser and a fraction of $\mu \mathrm{f}$ or thousands PF high frequency capacitance, showing in Fig. 4.

电流

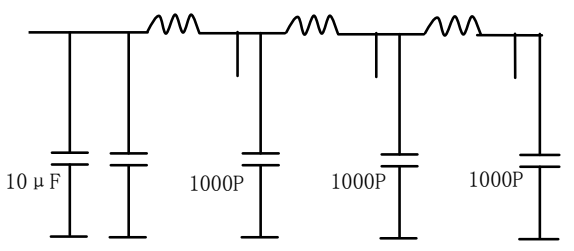

Figure 4. Filtering and decoupling circuit

The filtering and decoupling should be added in the power input and first-level source filter can also be added, showing in Fig. 5 which can help to improve order of magnitudes with excellent effect of inhibiting. Yet there is a shortage: the supply voltage reduction.

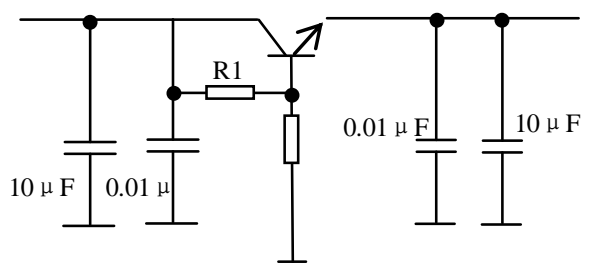

Figure 5. Active Power Filter

Proper power cord and feeder diameter should be selected to strengthen the shielding effect with twisted pair. The feeder line of the printing board should be wide enough to reduce the inductance and the characteristic impedance and weaken the noise interference. The trend of power cord should take order into consideration without closed cycle and the power cord should be shorter so as to avoid the damage of interference.

\section{B. Interference and electromagnetic protection measures for data system transmission}

Data transmission can be divided into two types. One is outside the data transmission line: when signal voltage and current are added to the transmission line there is an electric field generated. Main solution depends on the structure arrangement, proper wiring and shielding. 
Another is in the data transmission line: while there should be signal transmitting, there will be delay, reflection and radiation, especially the reflection, which will cause reflective jamming. The main approach is to solve matching and isolation.

Electromagnetic shielding is closely related to the structure design and wiring arrangement and we have to shield the sensitive sources and key combinations. While carrying out shielding, we should implement magnetic shielding and electric shield based on different conditions and both of them should take good ground connecting into consideration. Especially for sensitive important circuits such as crystal oscillator we have to strengthen or weaken the shielding effect. For sensitive circuits as well as interference source, we should properly add shielding measures such as low noise amplifier with high power gain, and the DC amplifier should be strongly shielded. Besides, the feed line with high-power, heavy current and high stability should be properly shielded. Meanwhile, we have to focus on the reasonability of the shielded wire so as to reduce the interference. Concerning the structure, the interference source should not be close to the sensitive circuit. Moreover, there should be reasonable arrangement wire. In terms of the internal routing measure, there is a problem of mutual induction which will lead to single interference and so that the distance of signal line should be far from each other without parallel and the wire should not be thin yet be short and close to the ground transmission. The long-line should adopt coaxial cable and reduce signal interference. Besides, the inlet to outlet of signal transmission should be straight. In terms of the digital integrated circuit, there are lowresistance devices with low voltage yet heavy current and therefore the interference is not obvious yet is significantly affected by the magnetic field. There is magnetic field coupling between the transmission signal and line and that is to say there is mutual inductance. Reducing the mutual inductance is the main means to prevent interference. Concerning the matching and isolation, while transmitting high frequency signal, the signal lie is treated as a long line and the reflection because of improper matching should be highlighted. Main measures to prevent the reflection are matching and isolation. In general, there is matching network and impedance transformer among various electric circuits and the input and output value should be $50 \Omega$ which can be matched with coaxial cable. Moreover we have to pay attention to the isolated area among electric circuits in terms of digital integrated circuit so as to guarantee the normal operation. Meanwhile, we have to pay attention to the pull-down and pull-up resistance and the matching resistance should be set in the receiving terminal. The characteristic impedance of twisted pair is among 120 $200 \Omega$ and if necessary we can connect with the matched load resistance.

The launching of certain data transmission system in certain channel will cause interference for the flight control system and the plane may lose control if the channel is used. Based on validating, if the launching channel is close the controlling channel of the operation system, the mutual inductance caused will ignite the change of current and lead to interference. Changing the cable louding and dealing with the coupling shall help to solve the problems which indicate that data transmission system cable laying and shielding shall be adapted to the overall compatibility of the plane.

\section{CONCLUSIONS}

The data transmission system asks hypo-phase shielding circuit and proper electromagnetic compatibility design or we cannot reach the expected target. The installation of data transmission system should have systematic structure design so as to guarantee the realization of data transmission capability. Shielding and module division should be adapted to electromagnetic compatibility design. What's more, the electromagnetic compatibility design of data transmission should have safe and reasonable circuit design which should be in accordance with the aircraft and product structure. No matter ground connecting setting, the cable distribution arrangement or the shielding and arrangement of electronic combination and material selection belong to electronic structure. The manufacturing of shielding layer, the installation of data transmission system will directly affect the shielding, radiation and transmission effect. However, existing technology cannot deal with the problem of electromagnetic compatibility, including the passive measure adopted to avoid the interference while launching the data transmission system by the Beacon.

\section{ABOUT THE AUTHOR}

Chen Zhenwei (1975.09.01-), male, born in Xifeng, Liaoning is an intermediate engineer who works in the military sales department of China Aviation Industry Shenyang Aircraft Corporation. He is mainly responsible for the debugging and testing of aircraft avionics system. He has completed various debugging and testing tasks for national key aircrafts and participated in electromagnetic radiation rsearch for military aircraft. Address: No.15 of 328 Mailbox Shenyang Aircraft Corporation, Lingbei Road, Shenyang, Liaoning Post code: 110850 E-mail: 1693706590@qq.com, Mobile phone: 13889146372.

\section{REFERENCES}

[1] Li Jiaqi, Zhao Xiaowang, Yang Shaokong. Aircraft Flight Test (2nd edition) [M] Edition department Domestic flight aviation

[2] Gu Dejun, Xia Zhenhua, Xu Caiju. Theory and technology of avionics equipment[M] Beijing: National Defense Industry Press, 2001 (1)

[3] Cui Yihua, Li Jikuan. Research for Spin Characteristics of Flight Test $[\mathrm{J}]$ Advances in Aeronautical Science and Engineering, $2012.2(3.1)$ 